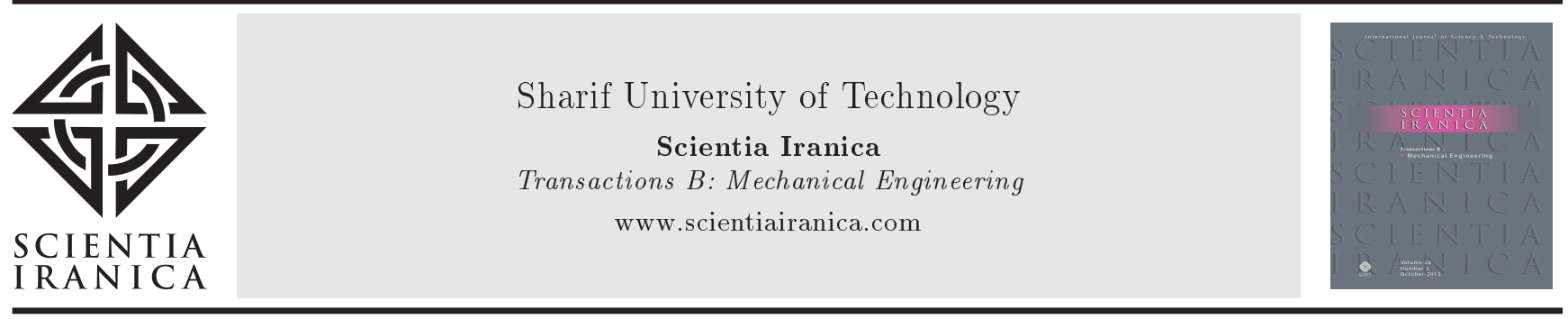

\title{
Modeling self-assembly of the surfactants into biological bilayer membranes with special chemical structures using dissipative particle dynamics method
}

\author{
S. Yaghoubi ${ }^{a, *}$, A.R. Pishevar ${ }^{a}$, M.S. Saidi ${ }^{b}$ and E. Shirani ${ }^{c}$ \\ a. Center of Excellence in Energy Conversion, Department of Mechanical Engineering, Isfahan University of Technology, Isfahan, \\ P.O. Box 8415683111 , Iran. \\ b. Center of Excellence in Energy Conversion, School of Mechanical Engineering, Sharif University of Technology, Tehran, P.O. \\ Box 11155-9567, Iran. \\ c. Department of Mechanical Engineering at Foolad Institute of Technology, Fooladshahr, Isfahan, P.O. Box 84915651, Iran.
}

Received 17 June 2013; received in revised form 5 April 2015; accepted 25 July 2015

KEYWORDS
DPD;
Biological membrane;
Lipid bilayer;
Flory-Huggins
parameters.

\section{Introduction}

The function of lipid bilayer membranes is extremely essential for living cells. They surround living cells and must be strong enough to protect their interior parts from the outside and also to prevent the penetration of unwanted ions and molecules. Because of special composition and dynamics, they have much more effective contribution to the considerable structural and

\footnotetext{
*. Corresponding author. Tel.: +98 3113915208 ; Fax: +983113912625

E-mail addresses: s.yaghoubikoupaye@me.iut.ac.ir (S. Yaghoubi); apishe@cc.iut.ac.ir (A.R. Pishevar); mssaidi@sharif.edu (M.S. Saidi)
}

\begin{abstract}
The aim of this study is to simulate the self-assembly of the surfactant odeled with special chemical structure and bending stiffness. To confirm that the novel model is physical, we determine the interaction parameters based on matching the (a) the relation between DPD parameters and of a lipid membrane, which is modeled by introducing a bond bending potential. To verify our model, we investigate the effect of surfactant structure, like chain length and stiffness of the molecules, on the properties of the modeled membrane as area per surfactant. To validate our results, we also compare them with the theoretical calculations as well as with
\end{abstract} (C) 2016 Sharif University of Technology. All rights reserved. material properties of cells, such as deformability in red blood cells [1]. Lipid bilayers systems behave extremely complex as they have a heterogeneous structure and dynamics and biological behavior of a wide range of spatial and temporal scales [2].

In biological lipid bilayers, the lateral pressure profile has an important function and it becomes the topic of discussions about the structure and role of proteins in membranes [3] or in the mechanism of anesthesia [4]. However, to determine the pressure profile for lipid bilayers, only the simulations or theories can be used, because there are no experimental results available [4]. Moreover, dynamic processes in a membrane occur on long distances compared to the molecular size, and on time scales much longer than 
the period of molecular vibrations. To investigate an all atom model of a membrane, it is possible to use molecular simulations and even some theoretical approaches. For example, lattice-based Monte Carlo (MC) simulations have been applied to consider the microstructures in systems containing water with the surfactants [5], and the structural properties of long or short lipid chains within a bilayer [6]. Meanfield technique is another example which is used by several groups to study the lipid bilayers [7]. Using this technique, they explore the dependence of membrane stability on some properties like the area per head group [8] and the length hydrocarbon tail [6]. Shillcock and Lipowsky [9] stated that "Both these approaches have certain limitations. Lattice based simulations lack the full Galilean invariance of a fluid, while mean-field theories ignore fluctuations within a system." Moreover, atomistic level methods such as Molecular Dynamics (MD) simulations have another limitation. They can be used for simulating systems with small sizes and short times [10]. Even with coarse-grained $\mathrm{MD}$, the computer time that is required to simulate a sizable membrane is too large. Therefore, a new particle-based mesoscopic simulation method has been used for the problem of simulating large membrane. The Dissipative Particle Dynamics was introduced for the first time in 1992 by Hoogerbrugge and Koelman [11]. Groot and Warren [12] modified this algorithm and used DPD method to study the phase separation of immiscible polymeric fluids. Espanol and Warren [13] introduced the fluctuation-dissipation theorem in this method.

Because of some advantages in using DPD method relative to other methods such as Molecular Dynamics (MD) or even coarse-grained MD simulations, we prefer this method to simulate the self-assembly of a model membrane. For example, since the potential function used in DPD method is softer than the potential used in $\mathrm{MD}$ simulations, the required time step in this method is significantly larger than the one used for the molecular dynamics simulations. Another challenge in simulating a biological membrane is choosing suitable boundary conditions. It is shown by Goetz and Lipowsky [14] that using periodic boundary conditions for simulating a bilayer leads to a membrane with a specified value of the surface tension. The amount of surface tension depends on the number of surfactants per area in the simulation. In our work, we used trial and error to make a membrane with zero tension. It is because of the state of biological membranes which are essentially tensionless [15].

Several groups have applied different methods to simulate self-assembly of the bilayer. For example, Goetz and Lipowsky in 1998 used Monte Carlo and Molecular Dynamics for simulating self-assembly of the bilayer and calculating the interfacial tension [14]. In 1999, Venturoli and Smit [16] simulated the selfassembly of surfactant bilayers using DPD method, but in their simulation, they did not use any special structure for their membrane.

Although in previous works, self-assembly of the surfactants into bilayer was modeled, in this work, we incorporate the surfactants with special chemical structure and bending stiffness, which makes our work distinct from the other studies. To this purpose, in practice, our model must agree with real physics. So, we find the interaction parameters based on matching the compressibility and solubility of the DPD system with real physics of the fluid. To match the mutual solubility for binary fluids, we use the relation between DPD parameters and $\chi$-parameters in Flory-Hugginstype models introduced in polymer chemistry. Eventually, to verify our model, we calculate the surface tension of the membrane to check to make sure it is tensionless as it is in real biological membranes. Also we investigate the effect of surfactant structure, like chain length and stiffness of the molecules, on the properties of the modeled membrane as area per surfactant. We observe that the location of the stiffness in the chains has significant effect on the properties of the bilayer. To validate our results, we compare them with the theoretical calculations as well as the experimental and other simulations results. We show that there is a good agreement between them.

The paper is organized as follows: The outline of dissipative particle dynamics method is briefly presented in Section 2; the required assumptions and simplifications to our model are discussed in Section 3; the equations that relate our simulations in DPD unites to physical unites are presented in Section 4; and in Section 5, the interaction parameters used in our simulations are described. Finally, the obtained results are given and discussed in Section 6 .

\section{Simulation methodology outline of the DPD method}

DPD is a particle-based method for simulating fluids that considers fluid elements as soft beads which interact via specified forces. A soft bead represents at least several molecules or molecular groups. With this coarse grained system to have a correct hydrodynamic behavior, soft beads must interact via effective forces. These pair wise additive forces conserve momentum and have no hard core. DPD is distinguished from Brownian Dynamics (BD) by use of momentum-conserving forces.

These forces include repulsion conservative force, dissipation force, and random force. All of these forces are effective within a certain cut-off radius, $r_{c}$. The force acting on a particle $i$ is then given by the following relation: 


$$
f_{i}=\sum_{j \neq i}\left(F_{i j}^{C}+F_{i j}^{D}+F_{i j}^{R}\right)
$$

where the sum runs over all other particles in a sphere with cut-off radius $r_{c}$. Similar to many other DPD simulations, we have used Eq. (2) for the conservative force [12]:

$$
F_{i j}^{C}= \begin{cases}a_{i j}\left(1-r_{i j} / r_{c}\right) \hat{r}_{i j} & \left(r_{i j}<r_{c}\right) \\ 0 & \left(r_{i j} \geq r_{c}\right)\end{cases}
$$

where $a_{i j}$ is the repulsive parameter or a maximum repulsion between particle $i$ and particle $j, r_{i j}=r_{j}-r_{i}$ and $r_{i j}=\left|r_{i j}\right|$. Dissipative or drag force and random force are given by [12]:

$$
\begin{aligned}
& F_{i j}^{D}=-\eta \omega^{D}\left(r_{i j}\right)\left(\hat{r}_{i j} \cdot v_{i j}\right) \hat{r}_{i j}, \\
& F_{i j}^{R}=\sigma \omega^{R}\left(r_{i j}\right) \theta_{i j} \hat{r}_{i j},
\end{aligned}
$$

where $\omega^{D}$ and $\omega^{R}$ are $r$-dependent weight functions which are equal to zero for $r>r_{c}, v_{i j}=v_{j}-v_{i}$, and $\theta_{i j}(t)$ is a randomly fluctuating variable with Gaussian statistics.

Español and Warren [13] showed that as the DPD thermostat consists of random and dissipative forces, the fluctuation-dissipation theorem must be satisfied:

$$
\omega^{D}(r)=\left[\omega^{R}(r)\right]^{2}, \quad \sigma^{2}=2 \eta k_{B} T .
$$

As a simple choice, we take:

$$
\omega^{D}(r)=\left[\omega^{R}(r)\right]^{2}= \begin{cases}(1-r)^{2} & (r<1) \\ 0 & (r \geq 1)\end{cases}
$$

It is assumed that all beads have the same mass $m$. We choose the particle mass, temperature, and interaction range as units of mass, energy, and length, respectively. Hence, $m=k_{B} T=r_{c}=1$, and for a time unit, we use the following expression:

$$
\tau=r_{c} \sqrt{m / k T} .
$$

In DPD, if the fluctuation-dissipation relation is satisfied, this method will produce correct values for $(N, V$, $T$ ) ensemble $[12,13]$. At each time step, the positions and velocities $\left\{r_{i}, v_{i}\right\}$ must be updated by the positions and velocities at earlier time using the modified version of the velocity-Verlet algorithm:

$$
\begin{aligned}
& r_{i}(t+\delta t)=r_{i}(t)+\delta t v_{i}(t)+1 / 2 \delta t^{2} f_{i}(t), \\
& \tilde{v}_{i}(t+\lambda \delta t)=\tilde{v}_{i}(t)+\lambda \delta t f_{i}(t), \\
& f_{i}(t+\delta t)=f_{i}\left(r_{i}(t+\delta t), \tilde{v}_{i}(t+\lambda \delta t)\right), \\
& v_{i}(t+\delta t)=v_{i}(t)+1 / 2 \delta t\left(f_{i}(t)+f_{i}(t+\delta t)\right) .
\end{aligned}
$$

If the parameter $\lambda$ is put at $\lambda=0.5$, this scheme will be the velocity-Verlet algorithm [17]. However, in the modified version of the velocity-Verlet algorithm, we use $\lambda=0.65$.

As mentioned above, we need to calculate the surface tension. The stress tensor leads to the surface tension via:

$$
\begin{aligned}
\gamma & =\int p_{x x}(x)-1 / 2\left(p_{y y}(x)+p_{z z}(x)\right) d x \\
& =A^{-1} \sum_{i<j}\left(F_{i j, x} x_{i j}-1 / 2\left(F_{i j, y} y_{i j}+F_{i j, z} z_{i j}\right)\right) .
\end{aligned}
$$

Suppose the bilayer is oriented perpendicular to the $x$-axis. Then, the local density of each component is measured in thin slabs perpendicular to the $x$-axis, and the stress tensor is averaged locally and over the whole system. Finally, in Eq. (8), $A$ is the area of the $y z$ plane, and $F_{i j}$ is the total conservative force between particles $i$ and $j$. In our work, we must measure $\gamma$ and control it to provide a tensionless membrane.

\section{Simulated system}

The simulated system includes water and some surfactant molecules with a special structure. Each surfactant contains one chain of hydrocarbons connected to a phosphate group. Thus, three types of particles are presented in the simulation: particles that model water $(w)$, the hydrophilic head $(h)$, and hydrophobic tail $(t)$ which are parts of the surfactants. The molecule with its mapping on the coarse-grained DPD model is shown in Figure 1.

For simplicity in this work, we have chosen methyl phosphate $\left(\mathrm{CH}_{2}-\mathrm{O}-\mathrm{PO}_{3} \mathrm{H}\right)$ as the head of the surfactant and every three $\mathrm{CH}_{2}$ as one tail bead. A surfactant molecule includes a linear chain of $h$ and $t$ units which are connected via harmonic springs based on the following relation:

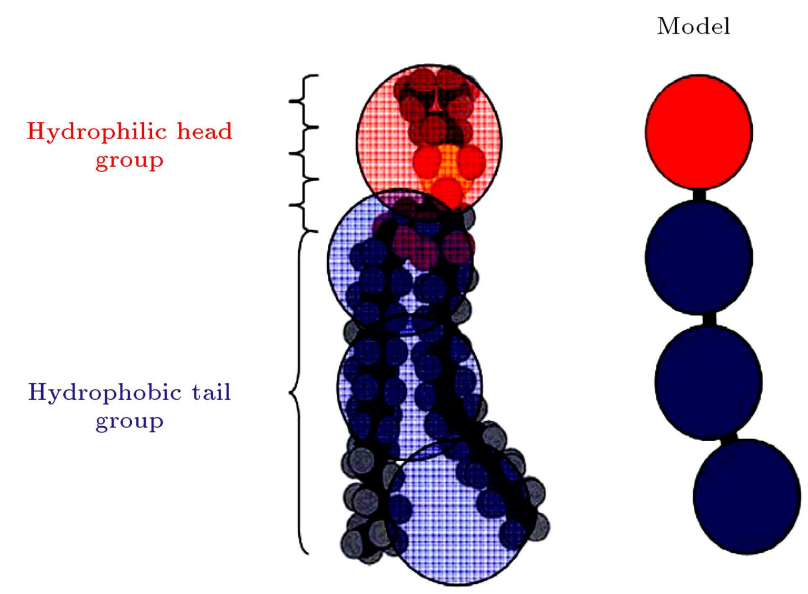

Figure 1. The simulated single chain and its mapping on the DPD model. 


$$
U_{\text {spring }}\left(r_{i, i+1}\right)=\frac{k_{r}}{2} r_{i, i+1}^{2},
$$

where $k_{r}=10$. This constant was chosen in order to have a bilayer with less surface tension. A linear chain with one head group and for example seven tail units is denoted by $h t_{7}$. The number of tail units will change and the effect of chain length on the bilayer's properties will be studied.

The stiffness of a lipid membrane depends on the unsaturated carbon bonds. Here, unsaturated bonds are modeled by introducing a bond bending potential to represent the stiffness of the chain like the following:

$$
\begin{gathered}
U_{\text {bend }}\left(r_{i-1, i}, r_{i+1, i}\right)=\frac{k_{\theta}}{2}\left(\theta-\theta_{0}\right)^{2} \\
=\frac{k_{\theta}}{2}\left(\frac{r_{i-1, i} \cdot r_{i+1, i}}{\left|r_{i-1, i}\right|\left|r_{i+1, i}\right|}-\theta_{0}\right)^{2},
\end{gathered}
$$

where $\theta$ is the angle between two successive bonds and the constants are set to $k_{\theta}=0.1$ and $\theta_{0}=\pi$. The presence of the bond bending potential is denoted by a capital $T$ for the $i$ th atom in the above equation. For example, $h t_{3} T t$ is a surfactant molecule which contains 6 beads with the bond bending potential in last three atoms of the tail. Both the Hookean spring and bondbending potential are parts of the conservative forces in a DPD simulation. In the following section, we describe the procedures used to encompass these potentials in the simulation.

\subsection{Hookean spring forces}

In this simulation, we assume that surfactant molecules are constructed by connecting $h$ and $t$ atoms via harmonic springs. Then, we exert Hookean spring force as a pair wise force between these two beads based on their positions.

\subsection{Bond bending forces}

If the bond bending potential in Eq. (10) is differentiated with respect to $\theta$, the bending moment on this bond will be achieved. Suppose the moment acts on a bond connecting particles $i$ and $i-1$, then the reaction moment is exerted on the adjacent bond connecting particles $i$ and $i+1$, as shown in Figure 2 .

The bond bending moment is obtained as:

$$
M=-k_{\theta}\left(\theta-\theta_{0}\right) \text {. }
$$

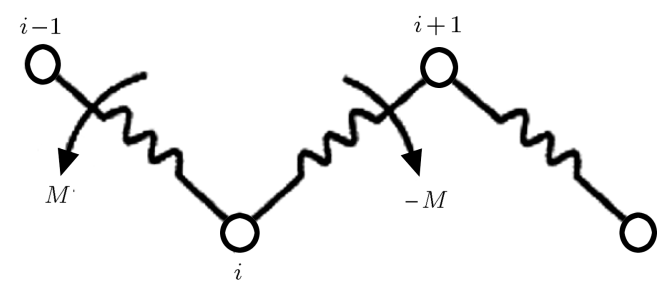

Figure 2. The bond bending moment in a pair wise manner.

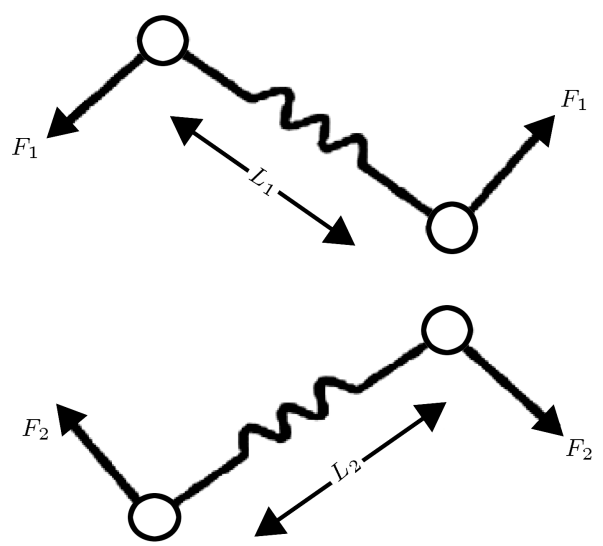

Figure 3. Illustration of replacing bond bending moment with coupled forces.

For the coding purpose, the moment is replaced by a pair of coupled forces. According to Figure 3, these forces are given as:

$$
F_{1}=\frac{M}{L_{1}}, \quad F_{2}=\frac{M}{L_{2}},
$$

where $L_{1}$ and $L_{2}$ are the distances between two adjacent particles and the bond bending moment is exerted on the spring between them. Since $M_{1}$ and $M_{2}$ are perpendicular to the plane containing particles $i-1, i$, and $i+I$, the equivalent pair wise forces, $F_{1}$ and $F_{2}$, are also exerted in the same plane.

\section{Physical length and time scales}

To construct a mesoscopic model and determine the length scale, at first, it is necessary to determine the volume of the simulated beads. We choose a coarsegraining particle, such as three carbon atoms which are supposed to be together into one bead. It is needed to justify the volumes of $\left(\mathrm{CH}_{2}\right)_{3}$ and water molecules. The volume of $\left(\mathrm{CH}_{2}\right)_{3}$ is $90 \AA^{3}$ and the water beads $(w)$ have to represent the same volume. As the volume of a water molecule is $30 \AA^{3}$, the water beads $(w)$ must represent three water molecules. Hence, each ( $w$ or $c$-) bead represents a liquid volume of $90 \AA^{3}$. We choose the bead density $\rho r_{c}^{3}=3$. It means a cube of $r_{c}^{3}$ contains three beads and then it equals a volume of $270 \AA^{3}$. Thus, we find the physical size of the cut-off radius as:

$$
r_{c}=\sqrt[3]{270} \AA=6.4633 \AA
$$

In general, if we take the coarse graining parameter as $N_{m}$ and suppose that a cube of volume $r_{c}^{3}$ represents $\rho$ beads, then the physical volume of this cube is $30 \rho N_{m} \AA^{3}$ and the length scale $r_{c}$ follows as:

$$
r_{c}=3.107\left(\rho N_{m}\right)^{1 / 3}[\AA] .
$$

We can calculate the reference mass for water as 
follows:

$$
m_{\mathrm{ref}}=m_{\mathrm{DPD}}=N_{m} \times m_{\mathrm{water}},
$$

where $m_{\text {water }}$ is the mass of each water molecule, which is calculated in our simulation with $N_{m}=3$ to be $3.9 \times$ $10^{-26} \mathrm{~kg}$.

The reference time is defined as the ratio of reference length $r_{c}$ to the reference velocity of the system and is given by the following ratio:

$$
t_{\mathrm{ref}}=t_{\mathrm{DPD}}=\frac{r_{c}}{u_{\mathrm{ref}}}
$$

For the reference velocity, however, many researchers have used the thermal velocity, $\sqrt{\frac{k_{B} T}{m_{\mathrm{DPD}}}}[13,18,19]$.

\section{Interaction parameters}

As it has already been mentioned, the bead density is fixed at $\rho=3$ and all the simulations have been performed at $k_{B} T=1$. Following Groot and Warren [12], a modified velocity-Verlet algorithm is used that allows us to use time steps of $d t=0.06 \tau$ and we also use the fixed noise amplitude $\sigma=3$.

In our simulations, we use 200 surfactants. The surfactant concentration $C_{s}$ is defined as:

$$
C_{s}=\frac{N_{h}+N_{t}}{N_{w}+N_{h}+N_{t}},
$$

where $N_{h}, N_{t}$, and $N_{w}$ are the numbers of heads, tails, and water particles, respectively. We set this parameter approximately equal to 0.39 for our simulations. At such density and concentration, the volume of the box has been chosen and a bilayer is formed.

To find the interaction parameters for this model, in practice, we need to match the compressibility and solubility of the DPD system with those in real physics. Following Groot and Warren [12], an equation is used in which the conservative interaction parameter $a$ is related to the thermodynamic definition of the isothermal compressibility $\kappa_{T}$ using the equation of state.

The isothermal compressibility is defined in a process where the temperature $T$ is constant as follows:

$$
\kappa_{T}=-\frac{1}{V}\left(\frac{\partial V}{\partial p}\right)_{T}=\frac{1}{\rho}\left(\frac{\partial \rho}{\partial p}\right)_{T},
$$

where $\rho$ is the bead density in the simulation. It is convenient to match the DPD system with real physics using the definition of the dimensionless parameter $\kappa^{-1}$ by:

$$
\kappa^{-1}=\frac{1}{k_{B} T \rho \kappa_{T}}=\frac{1}{k_{B} T} \frac{\partial p}{\partial \rho} .
$$

As the above parameter is a dimensionless parameter, we can use the following relation:

$$
\begin{aligned}
\left(\frac{1}{k_{B} T \rho \kappa_{T}}\right)_{\operatorname{sim}} & =\frac{1}{k_{B} T}\left(\frac{\partial p}{\partial \rho}\right)_{\operatorname{sim}} \\
& =\frac{1}{k_{B} T}\left(\frac{\partial p}{\partial n}\right)_{\exp }=\text { const. }
\end{aligned}
$$

Here, $n=N_{m} . \rho$ denotes the molecular number density of the physical system; "exp" denotes the experimental value, and the subscript "sim" refers to the simulation value. In general, while the DPD bead corresponds to $N_{m}$ of the water molecules, the system should satisfy:

$$
\begin{aligned}
\frac{1}{k_{B} T}\left(\frac{\partial p}{\partial \rho}\right)_{\operatorname{sim}} & =\frac{1}{k_{B} T}\left(\frac{\partial n}{\partial \rho}\right) \cdot\left(\frac{\partial p}{\partial n}\right)_{\exp } \\
& =\frac{N_{m}}{k_{B} T}\left(\frac{\partial p}{\partial n}\right)_{\exp }=\text { const. }
\end{aligned}
$$

where, $N_{m}$ is the number of water molecules per DPD bead.

The equation of state relates the pressure to the particle number density $\rho$. As Groot and Warren [12] mentioned for densities $\rho>2$ in reduced units (more than 2 particles in a box with length $r_{c}$ ), the following equation of state is a good approximation to the numerical simulations:

$$
p=\rho k_{B} T+a \alpha \rho^{2}, \quad \alpha=0.101 \pm 0.001 .
$$

From Eqs. (20) and (21) at constant temperature, we obtain the following relation:

$$
\frac{N_{m}}{k_{B} T}\left(\frac{\partial}{\partial \rho}\right)\left(\rho k_{B} T+a \alpha \rho^{2}\right)=\text { const. }
$$

The compressibility of water at room temperature is matched at 16 . Then, for $N_{m}=3$, the repulsion parameter in Eq. (2) must be equal to [12]:

$$
a_{i i}=78,
$$

where $i$ denotes the type of the particle. Following Groot and Rabone [20], the repulsion parameter between particles of the same type is taken the same for all liquid components; it is because the volumes of all liquid components are equal.

The next parameter which needs to be matched is the mutual solubility. In polymer chemistry, mutual solubility is specified by the Flory-Huggins $\chi$ parameters.

The Flory-Huggins $\chi$-parameters can be written in the following form:

$$
\chi=(0.231 \pm 0.001) \Delta a,
$$

where $\Delta a=a_{A B}-a_{A A}$ is the excess repulsion [19]. Using appropriate relations in polymer chemistry, the 
$\chi$-parameters are determined. They need to match relevant thermodynamic data to the same Flory-Huggins model. According to Groot and Rabone [20], we use the following parameters:

$$
\begin{aligned}
& \chi=\left(\begin{array}{cccc} 
& \langle w\rangle & \langle t\rangle & \langle h\rangle \\
\langle w\rangle & 0 & 6 & -0.5 \\
\langle t\rangle & 6 & 0 & 6 \\
\langle h\rangle & -0.5 & 6 & 2
\end{array}\right), \\
& a_{i j}=\left(\begin{array}{cccc} 
& \langle w\rangle & \langle t\rangle & \langle h\rangle \\
\langle w\rangle & 78 & 104 & 75.8 \\
\langle t\rangle & 104 & 78 & 104 \\
\langle h\rangle & 75.8 & 104 & 86.7
\end{array}\right) .
\end{aligned}
$$

Due to the above equation, we can specify the repulsion parameters based on Flory-Huggins $\chi$-parameters and relevant thermodynamic data. Therefore, we continue our simulations with a special chemical structure.

\section{Results, discussion, and verification}

Using the forces and parameters described above, we could construct a single surfactant molecule. In Figure 4, we illustrate the single surfactant molecule. For instance, we use $h t_{6}$ surfactants to examine the self-assembly. We start our simulations in the first step with a random distribution of surfactants (see Figure 5). The bilayer is formed for these surfactants after approximately 80000 iterations, as shown in Figure 6 .

The density profile for the bilayer with these surfactants consists of seven beads without any stiffness, (i.e. completely flexible), as presented in Figure 7. As it is clear, according to the diagram, the water molecules are completely out of the space, where occupied by the membrane (head and tail groups). Also, we see that the density of water molecules in this region is approximately 3 (the mean density of the simulation). Closer to the middle axis of the bilayer, it is obvious in the profile that the density of the water molecules decreases suddenly, although head groups converge more. It is because of hydrophilic head groups and their inclination to the water molecules. Further, near the middle axis, the tail groups converge as well.

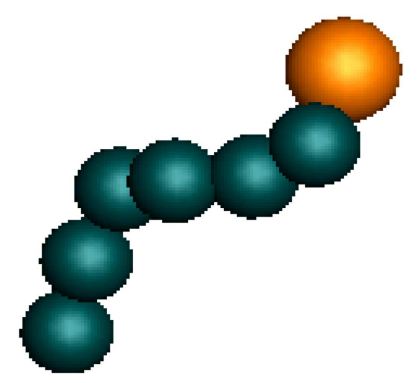

Figure 4. A single surfactant molecule, $h t_{6}$.
It is important to note that in both bilayer illustration and density profile (Figures 6 and 7), we see a symmetrical location for all kinds of DPD particles. This arrangement for gathering the particles in this simulation makes us confident about the results in

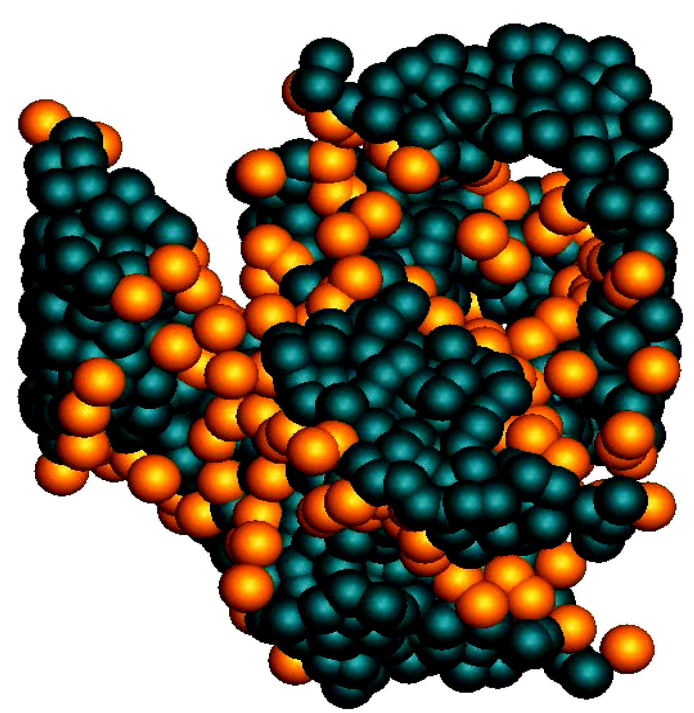

Figure 5. Initial configuration of the simulation of the self-assembly of a bilayer.

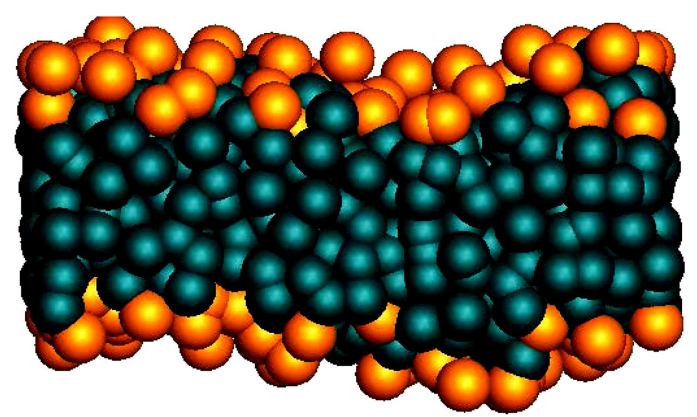

Figure 6. A bilayer starting from a random distribution of surfactants $\left(h t_{6}\right)$.

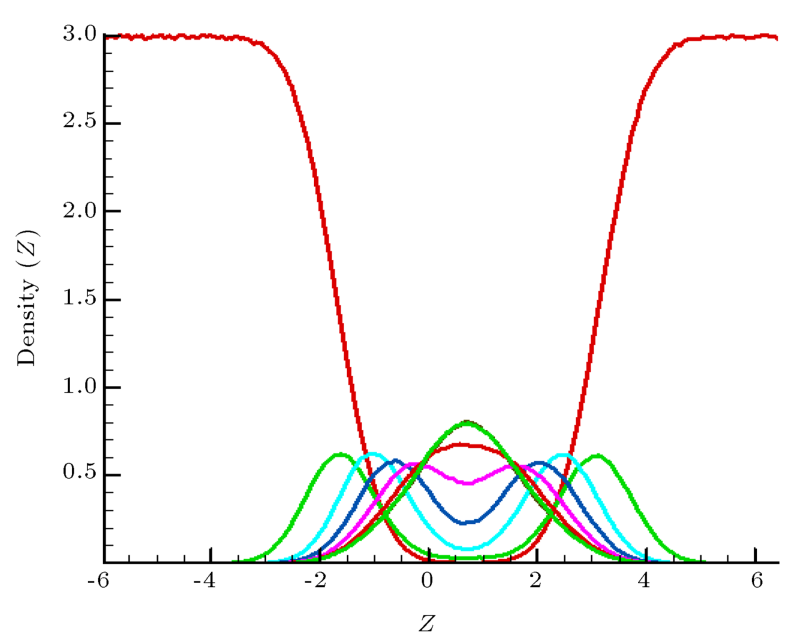

Figure 7. Density as a function of the distance from the middle of the bilayer $z$ axis for $200 h t_{6}$ surfactants. 


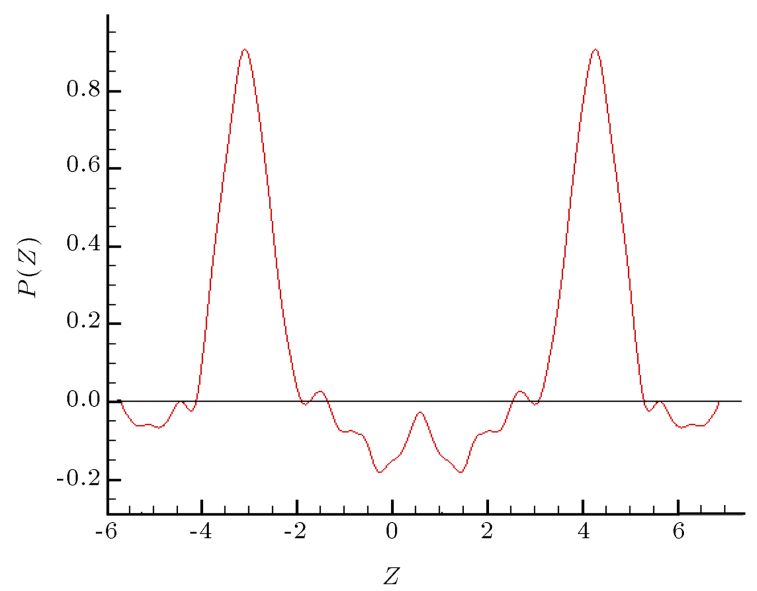

Figure 8. Pressure as a function of the distance from the middle of the bilayer $z$ axis for $200 h t_{6}$ surfactants.

simulating bilayer. These results are also in good agreement with that of Smit and Venturoli's [16]. They have simulated self-assembly of the surfactants with no special chemical structure. Also, our parameters qualitatively lead to the similar density profile to those reported by Groot [20]. According to the density profile in Figure 7 , we can evaluate the thickness of the bilayer. The thickness of this bilayer, with the properties mentioned in the text, is approximately equal to $4 r_{c}$. Note that we determined the amount of $r_{c}$ above.

The pressure profile for this flexible surfactant consists of seven beads, as presented in Figure 8. We define the pressure profile as the local surface tension:

$$
\gamma(Z) \equiv \Sigma_{T}(Z)-\Sigma_{N}(Z)
$$

where $\Sigma_{N}(Z)$ and $\Sigma_{T}(Z)$ are the normal and tangential components of the pressure tensor, respectively.

As it is a symmetrical profile, we add one-half of the pressure profile, symmetrically. Note that our simulation is for zero surface tension and the integral of this pressure profile is zero. We find a procedure for changes in the pressure profile versus $z$ axis very similar to what Goetz and Lipowsky [14] and also Smit and Venturoli [16] presented. It means that close to the head group, there is the first minimum in the pressure profile. If we move into the interior of the bilayer, we find that it is followed by a large maximum and in the middle of the bilayer there is a maximum again.

A very important criterion to validate the simulating membranes is the ratio of area per surfactant. Nagle and coworkers [21] pointed that the surface area of DPPC bilayers at $323 \mathrm{~K}$ is very close to $63 \AA^{2}$ per lipid. Our simulation leads to an averaged area of $64.7 \AA^{2}$ per lipid. In Comparison with [21] (which is listed literature values for area/lipid for different lipids, temperatures, and methods), we can also understand that our result is in good agreement with them.

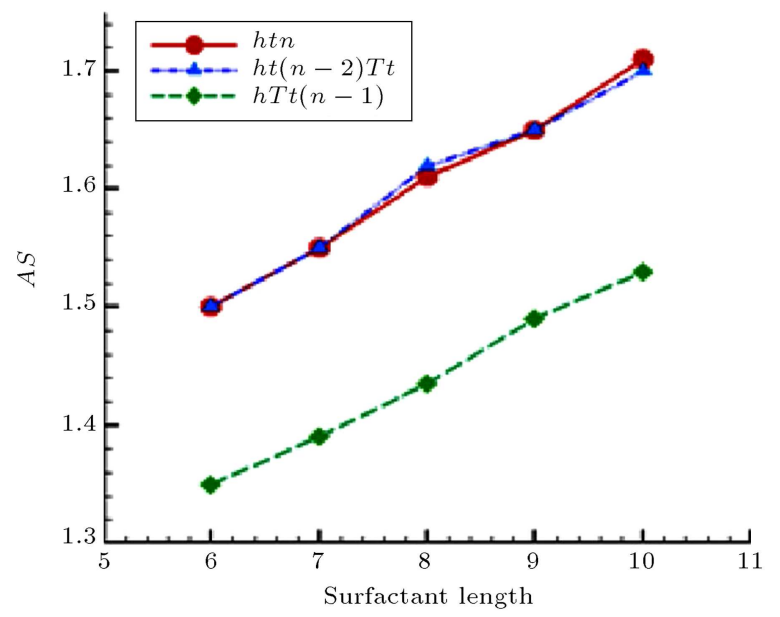

(a)

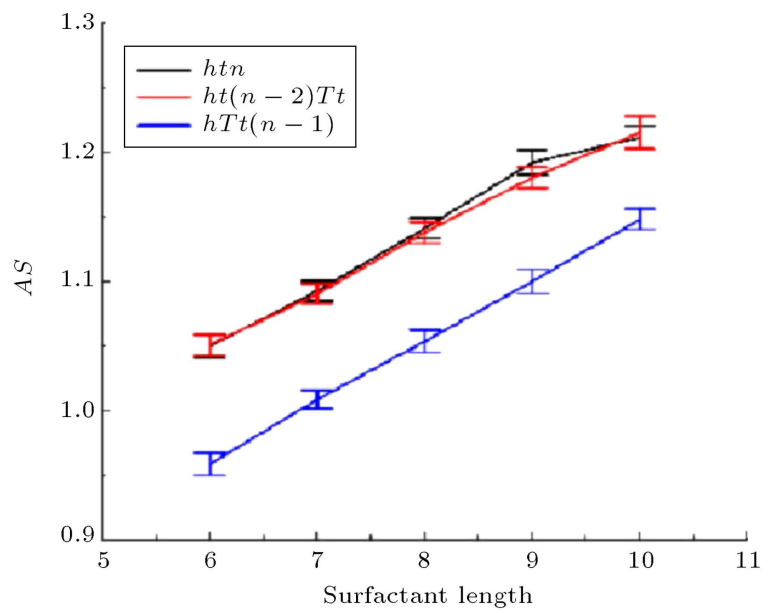

(b)

Figure 9. (a) Our simulation results. (b) Venturoli and Smit [16] results.

The influence of the tail length and stiffness on the average area per surfactant is shown in Figure 9. We compute the average area and divide this by half of the number of surfactants (because we have a bilayer) during the simulation to calculate the area per surfactant in this diagram. When the tail length increases, the area per surfactant is obviously increased. We compare our results with that of Venturoli and Smit [16]. They reported their membrane simulation and also the dependence of area per surfactant to tail length. As it is already explained, a significant difference between our simulation and Venuroli's work is the kind of membrane. In our study, simulation is based on a special surfactant with a specific chemistry structure. So, we find the interaction parameters based on matching the compressibility and solubility of the DPD system with real physics of the fluid. To match the mutual solubility for binary fluids, we use the relation between DPD parameters and $\chi$-parameters in Flory-Huggins-type models introduced in polymer chemistry. 
In Figure 9, we also show the effect of the stiffness on the area per surfactant. This figure shows that the effect of the location of the bond bending potential is considerable. As it is obvious in the diagram, when the potential is close to the head, the area per surfactant becomes smaller than those of the flexible chains. However, when it is located at the end of the tail, the area per surfactant is approximately similar to that of the flexible chains. The smaller the area per surfactant for potential near the head, the stronger ordering of the molecules and it results in a more compact structure for the membrane.

We see that our results are very similar to the Venturoli and Smit's simulations [16]. To compare these two simulations and to see the similarities, Venturoli and Smit's profile is shown in Figure 9(b).

To validate our results, we also compare the results with the theoretical calculations of Cantor [22]. These calculations were done on a lattice model. To have a similar length scale in all three studies, like Venturoli and Smit, we have scaled the areas in such a way to have the same area per surfactant for chains with length seven. Figure 10 shows that all studies predict that the area increases linearly with chain length and they have a very good correspondence to each other.

\section{Conclusions}

In this paper, we have shown that by using DPD method, we can simulate the self-assembly of bilayers. Also, we were able to give a chemical structure to our membrane and do this membrane simulation more real. So, we find the interaction parameters based on matching the compressibility and solubility of the DPD system with real physics of the fluid. To match the mutual solubility for binary fluids, we use the

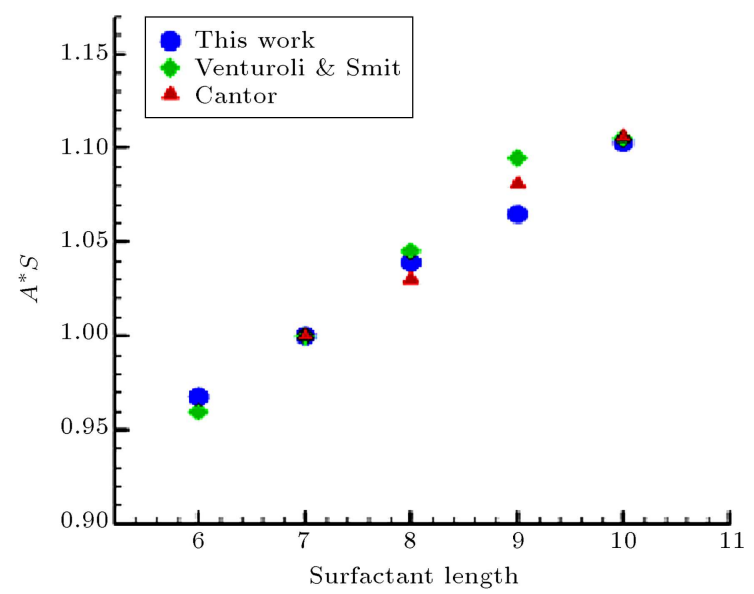

Figure 10. Our simulation results are comprised with Venturoli's work [16] and also the calculation of Cantor [22] considering completely flexible chains in a membrane. relation between DPD parameters and $\chi$-parameters in Flory-Huggins-type models introduced in polymer chemistry. It makes our work quite distinct from most of studies. Then, we validated our results with previous simulations and theoretical works. All studies predict that the area per surfactant increases linearly with chain length. We have also studied how the physical properties of the membrane were affected by changes in the structure of the surfactants. In addition to the tail length, the stiffness has a large effect on the bilayer properties, provided that this stiffness is located near the head group of the surfactants.

\section{References}

1. Alberts, B., Bray, D., Lewis, J., Raff, M., Roberts, K. and Watson, J.D., Molecular Biology of the Cell, 2nd Edn., Garland, New York (1989).

2. Lipowsky, R. and Sackmann, E., Structure and Dynamics of Membranes, 1st Edn., Elsevier (1995).

3. De Kruijff, B. "Lipids beyond the bilayer", J. of Nature, 386, pp. 129-130 (1997).

4. Cantor, R.S. "The lateral pressure profile in membranes: A physical mechanism of general anesthesia", J. of Biochemistry, 36, pp. 2339-2344 (1997).

5. Larson, R.G. "Self-assembly of surfactant liquid crystalline phases by Monte Carlo simulation", J. Chem. Phys., 91, pp. 2479-2488 (1989).

6. Harries, D. and Ben-Shaul, A. "Conformational chain statistics in a model lipid bilayer: Comparison between mean field and Monte Carlo calculations", J. Chem. Phys., 106, pp. 1609-1619 (1997).

7. Leermakers, F.A.M. and Scheutjens, J.M.H.M. "Statistical thermodynamics of association colloids. 1 . Lipid bilayer-membranes", J. Chem. Phys., 89, pp. 3264-3274 (1988).

8. Mueller, M. and Schick, M. "Calculation of the phase behavior of lipids", Phys. Rev. E., 57 6973, pp. 1-16 (1998).

9. Shillcock, J.C. and Lipowsky, R. "Equilibrium structure and lateral stress distribution of amphiphilic bilayers from dissipative particle dynamics simulations", J. of Chem. Phys., 117, pp. 5048-5061 (1992).

10. Marrink, S.J., Lindahl. E., Edholm. O. and Mark, A.E. "Simulation of the spontaneous aggregation of phospholipids into bilayers", J. Am. Chem. Soc., 123, pp. 8638-8639 (2001).

11. Hoogerbrugge, P.J. and Koelman, J.M.V.A. "Simulating microscopic hydrodynamic phenomena with dissipative particle dynamics", Europhys. Lett., 19, pp. 155-160 (1992).

12. Groot, R.D. and Warren, P.B. "Dissipative particle dynamics: Bridging the gap between atomistic and 
mesoscopic simulation", J. Chem. Phys., 107, pp. 4423-4435 (1997).

13. Español, P. and Warren, P.B. "Statistical-mechanics of dissipative particle dynamics", Europhys. Lett., 30, pp. 191-196 (1995).

14. Goetz, R. and Lipowsky, R. "Computer simulations of bilayer membranes: self-assembly and interfacial tension", J. Chem. Phys., 108, pp. 7397-7409 (1998).

15. Zhang, Y., Feller, S.E., Brooks, B.R. and Pastor, R.W. "Computer simulation of liquid/liquid interfaces. I. Theory and application to octane/water", J. Chem. Phys., 103, pp. 10252-102516 (1995).

16. Venturoli, M. and Smit, B. "Simulating the selfassembly of model membranes", Phys. Chem. Comm., 10, pp. 1-5 (1999).

17. Allen, M.P. and Tildesley, D.J., Computer Simulation of Liquids, Clarendon Press, Oxford, UK, pp. 78-81 (1987).

18. Revenga, M., Zuñiga, I. and Español, P. "Boundary conditions in dissipative particle dynamics", Comput. Phys. Commun., 121-122, pp. 309-311 (1999).

19. Pivkin, I.V. and Karniadakis, G.E. "A new method to impose no-slip boundary conditions in dissipative particle dynamics", J. Comp. Phys., 207, pp. 114-128 (2005).

20. Groot, R.D. and Rabone, K.L. "Mesoscopic simulation of cell membrane damage, morphology change and rupture by nonionic surfactants", Biophys. J., 81, pp. 725-736 (2001).

21. Nagle, J.F. and Tristram-Nagle, S. "Structure of lipid bilayers", J. Biochimica et Biophysica Acta., 1469, pp. 159-195 (2000).
22. Cantor, R.S. "Lipid composition and the lateral pressure profile in bilayers", Biophys. J., 76, pp. 2625-2639 (1999).

\section{Biographies}

Somaye Yaghoubi obtained her BS and MS degrees at Isfahan University of Technology, Iran, and is currently a PhD student of Mechanical Engineering at Isfahan University of Technology, Isfahan, Iran. Her research interests are biofluid simulation, modeling of particle transport, and CFD simulations.

Ahmad Reza Pishevar is Professor of Mechanical Engineering at Isfahan University of Technology, Iran. He is Editor-in-Chief of the Journal of Computational Methods in Engineering. His research interests include compressible unsteady flow, multiphase flows, and CFD simulations.

Mohammad Said Saidi is Professor of Mechanical Engineering at Sharif University of Technology, Tehran, Iran. His research interests include modeling and numerical analysis of transport and deposition of aerosol particles, modeling and numerical analysis of biofluids, modeling and numerical analysis of thermalhydraulics of porous media and microchannels.

Ebrahim Shirani is Professor of Mechanical Engineering at Isfahan University of Technology, Iran, and also at Foolad Institute of Technology, Fooladshahr, Isfahan, Iran. He is Editor-in-Chief of the Journal of Applied Fluid Mechanics. 